Thierry Karsenti Université de Montréal

\title{
La formation pratique des enseignants : regards croisés sur l'Afrique, l'Amérique et l'Europe
}

Michel Lepage Université de Montréal

\section{Chroniques internationales spécial formation pratique}

Les dernières décennies ont vu apparaître des changements majeurs dans les pratiques d'enseignement et dans les paradigmes qui les sous-tendent. Comment la formation des enseignants, et en particulier la formation pratique, a-t-elle su répondre aux attentes des nouvelles approches préconisées? Réunis autour de thèmes unificateurs sur la formation pratique des enseignants, les participants à ce symposium tenteront de tracer les grandes lignes des principaux axes privilégiés par la formation des enseignants du primaire et du secondaire dans leur système d'éducation respectif. Les types de parcours proposés aux futurs maitres ainsi que les liens théorie-pratique mis de l'avant dans leur formation seront présentés. Une place importante sera faite à l'organisation générale des stages et aux éléments liés à l'accompagnement des stagiaires, au choix et à la formation des accompagnateurs, à leur formation ainsi qu'à leur rôle dans l'évaluation. Dans ces chroniques de Formation et profession, nous avons cherché à réunir des textes de chercheurs et de praticiens de trois continents dans le but d'encourager des échanges qui permettront de faire ressortir les principaux atouts des divers types de formation pratique, mais aussi les défis auxquels ils sont confrontés. 